\title{
A New Social and Technological Paradigm to Assess Chronic Patient Management Process: Preliminary Results
}

\author{
Marco Benvenuto, Carmine Viola \\ University of Salento, Salento, Italy \\ Francesco Sambati \\ Universus CSEI Consortium, Bari, Italy
}

\begin{abstract}
The present research intends to address in a comprehensive, transversal, and interdisciplinary manner the chronic patient management process in the research project named "PRO DOMO SUD” in order to identify operational inefficiencies, thus demonstrating that these are largely attributable to incurred costs and, thus, evaluate possible solutions for providing effective and appropriate responses by healthcare and social services. Can patients/older people be treated, monitored, and managed successfully with mobile and wearable technologies? The project involved three different groups of patients/participants: Patients with heart failure shock in "Home Monitoring Scenario"; Patients with different pathologies in "Virtual Ward Scenario"; Patients with limited mobility due to Neurological and Orthopaedic disease in "Rehabilitation Scenario". Due to the complexity of the issue, the methodological approach adopted must be multidimensional and interdisciplinary, addressing the complexity of the chronic patient from all viewpoints, not reducing it, yet analysing, understanding, rearranging, and managing it in an organic manner. The three different scenarios were allowed to identify several impacts on organizational and clinic management of chronic diseases, the tests showed significant improvements in quality of life of patients enrolled in the project. The data deriving from the three scenario demonstrate that wearable divide and ICT, in general, can empower both patients and physician personnel allowing them to be active part in the chronic disease management process. The PRO DOMO SUD experience derived from the Living Lab, this is a new paradigm for industrial research and development activities which allows the final users to actively collaborate with the designers and technicians in the development and test of new products and services aimed to them. The Living Labs stimulate social innovation by transferring research results from the closed industrial laboratory towards real life contexts where citizens and users become co-developers.
\end{abstract}

Keywords: co-creation, cost, efficiency, HTA, chronic patients monitoring, home monitoring, hospital monitoring, social innovation

\footnotetext{
Acknowledgment: This work has been cofounded by P.O. FESR PUGLIA 2007-2013-ASSE I-Linea di Intervento1.4-Azione 1.4.2— “Investiamo nel vostro futuro”. The authors thank ESS—Rete per la Sanità Digitale, composed by Evolvo srl, Sepi Spa and SDS srl. Particular acknowledgement is due to the clinical center Fondazione San Raffaele Cittadella della Carità and the research center ISBEM-Istituto Scientifico Biomedico Euro-Mediterraneo.

Marco Benvenuto, professor, Department of Economics, University of Salento, Salento, Italy.

Carmine Viola, professor, Department of Economics, University of Salento, Salento, Italy.

Francesco Sambati, Mr., Universus CSEI Consortium, Bari, Italy.

Correspondence concerning this article should be addressed to Marco Benvenuto, University of Salento, Ecotekne Build. C-6, Lecce, Italy.
} 


\section{Introduction}

Average life expectancy in Europe is one of the highest in the world. By 2050 it will be, according to Eurostat, 79.7 years for men and 85.1 years for women. The percentage of the European population aged over 65 is expected to rise from $16.1 \%$ in 2000 to $22 \%$ by 2025 and $27.5 \%$ in 2050 with the population aged over 80 (3.6\% in 2000) estimated to reach 6\% by 2025 and $10 \%$ by 2050 (D. Bloom, Cafiero, Jané-Llopis, Abrahams-Gessel, L. Bloom, Fathima, Feigl, Gaziano, Hamand, Mowafi, O’Farrell, Ozaltin, Pandya, Prettner, Rosenberg, Seligman, Stein, Weinstein, \& Weiss, 2012).

On the basis of such data, chronic diseases closely related to the ageing population represent the main issue for public health according to the WHO. The ageing associated with the increasing demand for high a quality of life puts National Health Systems under major management and economic pressures due to the increasing prevalence of poly-chronic patients who are associated with issues of higher costs (WHO, 2010). The challenge for the near future is to be able to define appropriate management and technology systems that take into account such complexity.

However, despite the pathway seems clear, easy, and immediate, a large debate still exists among economists, physicians, policy makers, and researchers to find the causes of the failure of a widespread and systematic diffusion of ICT in healthcare services: legal issues, organizational models, lack of systematic diffusion planning, lack of reliable cost effectiveness data (Cunningham, 2010). The concept of the following research is based on the belief that many expenditure determinants are due to issues and inefficiencies related to patient management. In addition to clinical issues, there is a managerial dilemma related to dimensions and aspects sometimes directly dependent on health care organizational models-e.g. hyper or multi prescriptions for diagnostic tests or incompatible drugs-sometimes resulting from dimensions often considered negligible- e.g. the social dimension—with a direct impact on health outcomes (Paulden \& Claxton, 1993; Fried, Lovell, \& Schmidtet, 1993).

The paper is organized as follows: first, a review of previous literature on chronic patient management through Telemedicine is presented. The project objectives and research methodology are described. Finally, the results and discussions concerning implications of PRO DOMO SUD project and research on chronic patient management are presented.

\section{Literature Review}

Telemedicine refers to a range of services that can be administered through the application of Information and Telecommunication Technologies (ICT). It represents a particular way of providing healthcare, through which medical resources, both basic and specialist, are available to patients who can not directly access to health services for various types of difficulties. The use of advanced telecommunication systems makes it possible to transmit medical information from the patient to the healthcare facility, and vice versa, avoiding physical displacement (Weinstein, Lopez, Joseph, Erps, Holcomb, Barker, \& Krupinski, 2014).

Telemedicine services should therefore be characterized by the same benefits as are required for a hospital: the continued presence of medical and paramedical staff, the availability of diagnostic, therapeutic and remote monitoring equipment, and timely reaction capability to a risk or emergency situation. Telemedicine in fact consists of a set of means and forms of intervention aimed at providing the assistance provided in decentralized structures compared to hospitals, while maintaining or even improving the quality of the service. 
Out-of-hospital care relies on efficiencies and efficiencies in distance communication between assistants and medical centers: only in these conditions can physicians intervene remotely in different types of situations, determining the appropriate actions to follow patients in remote (Pacht, Turner, Gailiun, Violi, Ralston, Mekhjian, \& St John, 1998). Networks, systems, and telecommunications services are obviously indispensable in evolutionary scenarios where remote monitoring and management projects are implemented (Marcin, Nesbitt, Cole, Knuttel, Hilty, Prescott, \& Daschbach, 2005). It is therefore crucial that structures are increasingly at the forefront of technology and the staff with the devices in use so that it is possible to establish remote relationships between patients and caregivers.

The complexity of clinical and healthcare management developments is a fertile field for the development of telemedicine technologies. In particular, the increase in the elderly population, the greater frequency of chronic clinical conditions, and the development of diagnostic and therapeutic profiles represent a challenge and an opportunity for the field of Telemedicine.

The management of the long-term condition of chronic patients has discussed the architecture of the National Health System, which provided for the provision of care only through the central figure of the hospital, and has proven it economically, with inefficient resources distribution available. The enormous political and economic pressure to reduce the costs of healthcare, pushes for the slower therapeutic protocols and the reduction of hospitalization, which is universally recognized as one of the main determinants of high costs of healthcare spending. In front of chronic diseases, in fact, hospitals are not always the best solution, especially when they have stabilized.

The main purpose of developing complex and integrated telemedicine systems is to evaluate the feasibility and usability of telemedicine and telemonitoring of patients with various chronic conditions. Patient's involvement in self-care is the keystone that allows continuous attention to the condition of the subject. A chronic illness effectively supported and educated by specialized staff is able to evaluate his or her health status, be independent in measuring and remotely transmitting its parameters, and then becomes a reliable interlocutor at crucial critical risk management moments. But to achieve this, three steps must be taken in the direction of Telemedicine: the first concerns health professionals who need to re-update their professionalism and redesign their roles, secondly, hospital companies that need to have adequate IT and telematic infrastructures, and finally the third who declines with the understanding of the patient's needs and limits (Pinnock, Hanley, Lewis, MacNeed, Pagliarie, Van Der Polf, Sheikhg, \& McKinstryh, 2010).

From studies in literature (Basilakis, Lovell, Redmond, \& Celler, 2010; Pinnock et al., 2010) are evident how fundamental it is to redesign National Health Systems in view of the ever-increasing need to manage chronic and multi-pathological patients. Indeed, the impact of these diagnostic-therapeutic profiles on economic terms is high. The services offered to chronic patients must therefore be redesigned around the patient, not the individual pathology, with a particular focus on prevention, technological tools, and self-management. In addition, Telemedicine was seen as an effective means of distributing health services even in isolated and remote communities (Lovell, Redmond, Basilakis, Shany, \& Celler, 2010). Telemedicine offers the possibility of drastically reducing unnecessary specialist consultations and delays, due to the large distances to be filled, on communication that is delayed in implementing the modifications of treatments and therapies. It enables, in fact, the transmission of remote diagnostic examinations, remote consultation with specialists, the widespread diffusion of specialist care and an evolution of the relationship between personal to the socio-sanitary sphere and the assisted. In addition to the territorial expansion of the systematic use of specialist skills, the 
development of Telemedicine allows a better qualitative distribution of healthcare and a better cost-benefit ratio, especially bearing in mind the reduction of all those inpatients is considered useless because it was essentially determined by an inability to independently manage the exacerbations of chronic illnesses. Telemedicine equipment and telemonitoring services therefore allow an interactive relationship between a general practitioner and a specialist physician, including nurses, both inside and outside healthcare facilities, as well as between these professional and patient due to the transmission of biomedical signals.

In conclusion, the analysis of literature has revealed the fundamental nature of teamwork and interdisciplinary collaboration in order to cancel times and distances between patients and healthcare structures. The aim of Telemedicine is therefore to integrate telematics and medical professionalism in such a way that, even from remote locations, it is possible to benefit from specialized resources physically allocated to centralized locations. This virtually shortens the gap between the "home" patient and the medical service provider. Through computer tools, audiovisual contact between physician and patient becomes possible, control and setting of device operation used and real-time acquisition of parametric clinical and instrumental devices such as blood pressure, body temperature, oxygen saturation and electrocardiogram.

\section{Materials and Methods}

\section{Outlines of the Project}

Activity started by proposing PRO DOMO SUD, a regional research project focused on ICT solutions for healthcare assistance services addressed to frail and elderly people. The project consortium was composed of (i) three SMEs operating in the field of ICT for healthcare, (ii) a clinical and rehabilitation center for cardiologic and orthopedic disease, and (iii) a biomedical research center.

During the project there were implemented and tested three solutions for the management, evaluation, and treatment of patients/older people with mobile and wearable technologies. Specifically, the three activities are (i) the home monitoring of chronic patients (mainly heart disease), (ii) in-hospital monitoring of geriatric patients, (iii) the evaluation of patients in rehabilitation through the using of quantitative protocols and sensors (Patel, Park, Paolo, Chan, \& Rodgers, 2012). Therefore, it implemented an ICT platform to manage in an integrated manner these three use cases, with the idea of achieving a sufficiently modular and open system that can allow in the future the extension to further medical management scenarios. For each of the three activities identified, a set of hardware interface devices have been identified and/or developed with the common information platform intended for different types of users. The following list outlines the characteristics of the activities:

\section{Home monitoring}

(a) Type of patient: chronic heart disease;

(b) Hardware used: smartphone Android, blood pressure monitor, pulse oximeter, bathroom scales;

(c) User Type: patient and general practitioner;

(d) Data used: systolic and diastolic blood pressure; mean arterial pressure; heart rate; oxygen saturation (\%), weight.

\section{Virtual Lane}

(a) Type of patient: long-stay elderly patient;

(b) Hardware used: tablet;

(c) User Type: nurse and doctor;

(d) Data used: systolic and diastolic blood pressure; mean arterial pressure; heart rate; oxygen saturation 
(\%); data nursing; clinical data derived from medical records.

3. Motor rehabilitation

(a) Type of patient: patient trauma or orthopedic prosthesis;

(b) Hardware used: wearable wireless sensors;

(c) User type: patient, physiatrist and physical therapist;

(d) Data used: range of motion hip and knee, six minute walking test.

The objective was to evaluate and quantify the contribution that innovative technologies and/or consumer can determine from a clinical, logistical, and operational management point of view with respect to the traditional management of the chronic patient as reported below (Claxton, Paulden, Gravelle, Brouwer, \& Culyer, 2011).

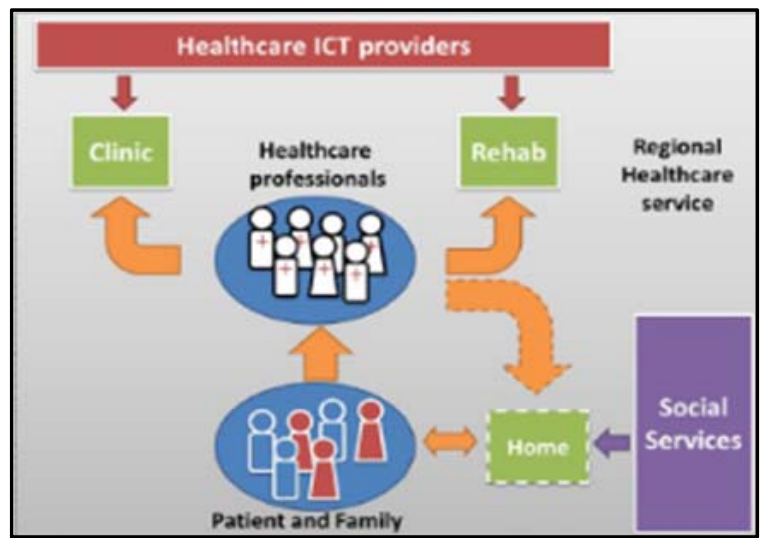

Figure 1. The organizational model of traditional healthcare services in Italy, from which the proposed project started.

Figure 1 draws schematically a simplified model of the current interactions for a typical patient with cardiologic diseases: acute patient who requires specific and urgent care goes to the clinical center (through emergency services or by the family) and gets hospitalized. Once he receives the treatments, he goes through a rehabilitation process (if needed) or he is directly discharged and returns home (Clark, Conway, Poulsen, Keech, Tirimacco, \& Tideman, 2013). Often, especially in cases of chronic disease, continuous but simple care and monitoring should be provided to the patient, allowing an early intervention on the patient in case of exacerbation of the problem. An early intervention would avoid a new hospitalization, thus reducing the cost for the welfare and increasing the Quality of Life for the patient (Devlin \& Parkin, 2004).

The identification and selection of the three lines of experimentation followed a rational mind: they showed three possible application scenarios of health care that could most benefit from technological systems of consumer with different purposes such as improving clinical outcomes, streamline charges or objectify, systematize and integrate the data health.

\section{Results}

In the first scenario the main innovation is focused on organization and clinical healthcare services: in the traditional model of care, patient management in the territory is delegated to the general practitioner (GP) who must take responsibility for ensuring coverage all his patients. In the model proposed by PRO DOMO SUD, technology and services developed, suitably designed with power users and cooperative patients, allow a more accurate parallel management particularly suitable for chronic patients, in which they play an active role in 
participating to the process; a team of suitably trained professionals offer services complementary to those provided by traditional territorial medicine (Simoens, 2010). In this way, while retaining decision-making, autonomy and centrality in the activity (Billis, Papageorgiou, Frantzidis, Tsatali, Tsolaki, \& Bamidis, 2015), the GP can make use of an auxiliary service consisting in punctual and daily warns only in cases of problems.

The second scenario instead involves the use of a technological system for the digital management of data relating to geriatric hospitalized patients.

The system is accessible and available via web and has the possibility of data entry through portable devices (tablet). The platform provides the management of health medical and nursing data, allowing consultation and printing of integrated reports. In this case, the innovation regards internal healthcare processes, in which the traditional method of managing and accessing data is replaced by digital technologies, achieving dematerialization and ubiquitous access.

Finally, the third scenario involves innovative technological aspects: an integrated system consisting of wearable wirelessly connected bracelets capable to detect the orientation of the parts of the body they are hooked to, has been developed. The goal is to make the measurements necessary to assess the physical condition of the patient in rehabilitation more objective and repeatable, thus improving the outcome of the rehabilitation process. The system allows the detection of the maximum and minimum joints angles (range of motion) of orthopaedic patients with prostheses (hip and knee), but also of neurological patients (Moeslund \& Granumet, 2001). A specific protocol was also defined and implemented for the quantitative evaluation of a functional test named Six Minute Walking Test able to determine the capacity of mobility of the patient (Kakadiaris \& Metaxas, 2000).

\section{First Scenario: Home Monitoring}

The first experimental line shows how the simple monitoring of physiological parameters, daily collected at home autonomously by the patient with chronic disease, properly integrated with customer care services and providing technical and logistical medical personnel remote access to clinical data patient, entails a likely reduction in spending on health care and an increase in quality of life of the patient (Figure 2). The complexity in the testing was to conceive an innovative model of interaction between the actors involved (Ward, 2013): patient, GPs, specialist doctors, nurses, employees, etc.

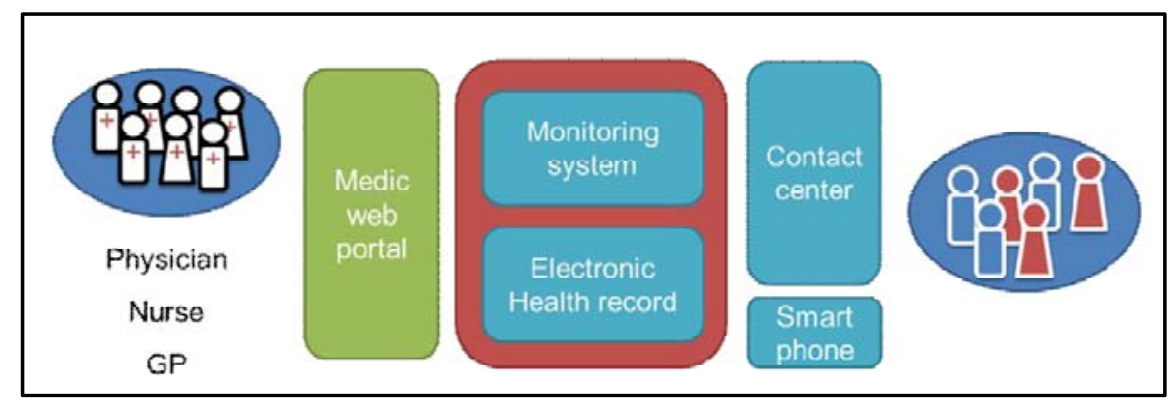

Figure 2. PRO DOMO SUD schematic model of home care..

The objective of this phase of experimentation is to reduce the occurrence of acute events that would lead to recurrent patient hospitalization. This is a win-win approach where you get the benefit from the quality of life of patients and cost savings for the National Healthcare System.

From the patient archive of the clinical center, a first set of patients were identified to be enrolled for the 
trial on the basis of three main parameters: (i) high number of accesses to the structure during the year 2013, (ii) the overall clinical status of the patient, (iii) age. Finally, 10 patients were successfully contacted and enrolled. The selected sample has thus the following features exposed in Table 1.

Table 1

Sample Description

\begin{tabular}{ll}
\hline Numerosity & 10 \\
\hline M/F & $80 / 20 \%$ \\
Age & 68.9 years \\
$\mathrm{N}^{\circ}$ of hospitalizations for heart failure average (2013) & 2.8 \\
Health spending average in 2013 (hospitalization) & $€ 15,123.33$ \\
\hline
\end{tabular}

First of all, 11 patients were recruited in total, because during the experimental phase there was a case of drop-out, which was promptly replaced with another suitable patient. However, since the dropout occurred in the final part of the monitoring period, the analysis was performed on 10 subjects. The average age is quite high, in the range of minimum 49 and maximum 81 years. The autonomous daily recording of the measures by the patients (without the collaboration of technical staff or nursing) implies that the interface designed and implemented in the platform was simple and functional enough, especially considering the advanced age of the sample. Note that the recruited patients were hospitalized for "heart failure and shock" an average of 2.8 times during 2013, ranging from minimum two times to maximum six. It is noteworthy that although theoretically the (Diagnosis Related Group) DRG reimbursement for hospitalization for "heart failure and shock" is slightly less than $€ 3,000$, the actual average cost for each patient was approximately $€ 5,400$, including also subsequent cardiac rehabilitation required. On average, therefore, each patient costs the National Health Service (NHS) over €15,000.

The sample described above was subjected to a campaign of monitoring of physiological parameters (blood pressure, heart rate, blood oxygenation, body weight) recorded each day and independently by patients. To ensure technical and logistical assistance to the patient a call center service with properly trained operators was created. The service contacted each patient at least once a week to ensure the positive trend of the trial and check for any problems (Zanaboni \& Wootton, 2012).

As can be seen from the graph (Figure 3), the phone calls were classified by topics into three main classes. The first has been named "Routine" and includes the cases where the patient manifested neither a technical, nor a clinical issue. As you can see, this category is equivalent to $70 \%$ of total calls. Technical problems, including any sessions of technical support, are included in the category "Technical problem", which makes up about a quarter of total calls. Finally, 6\% final, is the call, in which there was a potentially problematic event and a clinical alarm was reported.

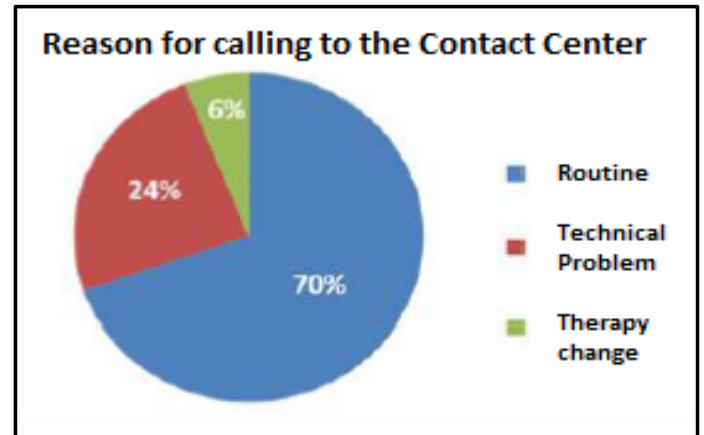

Figure 3. Topic of the calls between patients and the contact. 
The contact center service has been much appreciated by the patients, who felt pushed to a more proactive participation in the trial. This assessment has been extracted from a customer satisfaction questionnaire given to end users, as also required by the guidelines on telemedicine.

In order to standardize all fragmented experiences about telemedicine in the country, the Ministry of Health released in February 2014, the Guidelines on the Telemedicine (Ministero della Salute, 2014); in this document there is a specific section dedicated to the performance indicators of projects and experiences, useful to report a series of conventional parameters defining common criteria evaluation. In the evaluation of the project PRO DOMO SUD these indicators were tested (Table 2), well aware that some of these have no values because PRO DOMO SUD is a pilot trial and not a telemonitoring service in standard use. In particular, the main limiting factor is the duration of monitoring period (eight months).

Table 2

Indicators From Guidelines on the Telemedicine Applied on PRO DOMO SUD Project

\begin{tabular}{|c|c|c|}
\hline Indicators & PRO DOMO SUD & Notes \\
\hline \multicolumn{3}{|l|}{ Dimension } \\
\hline Number of patients observed in 12 months & 10 & The testing last 8 months \\
\hline $\begin{array}{l}\text { Number of patients observed in } 12 \text { months/number } \\
\text { of patients observed in the previous } 12 \text { months }\end{array}$ & & First year of testing \\
\hline Coverage of target (\%) & $0.14 \%$ & $\begin{array}{l}\text { Target }=\text { patients hospitalized for heart } \\
\text { failure }\end{array}$ \\
\hline Average number of contact/month & 36.14 & \\
\hline \multicolumn{3}{|l|}{ Temporal continuity } \\
\hline Number of months of activity since start up & 8 months & \\
\hline Dispersion index & & Period of testing too short \\
\hline \multicolumn{3}{|l|}{ Complexity } \\
\hline Expert involved & $\begin{array}{l}\text { Phone dialer trained, healthcare } \\
\text { assistant, specialist, general doctor }\end{array}$ & \\
\hline Number of operator involved (MP/users) & $(1+0.125+0.05) / 10=0.1175$ & See infra \\
\hline \multicolumn{3}{|l|}{ Quality } \\
\hline Standard time of reply & $<24 \mathrm{~h}$ & $\begin{array}{l}\text { Reply to a issue promoted by a patient } \\
\text { or detected }\end{array}$ \\
\hline \multicolumn{3}{|l|}{ Efficiency } \\
\hline $\begin{array}{l}\text { Cost of maintenance of service/number of patient } \\
\text { observed }\end{array}$ & Approx. 600€/yr/patient & \\
\hline \multicolumn{3}{|l|}{ Effectiveness } \\
\hline Death rate reduction & & No control group, no death recorded \\
\hline Hospitalization request reduction & & $\begin{array}{l}\text { No control group, no hospitalization } \\
\text { request recorded }\end{array}$ \\
\hline Emergency service request reduction & & $\begin{array}{l}\text { Non control group, one emergency } \\
\text { service request recorded }\end{array}$ \\
\hline Improvement in quality of life & & Not detected \\
\hline \multicolumn{3}{|l|}{ User satisfaction survey } \\
\hline Drop-out/12 months & 1 & \\
\hline Drop-out/12 months/number of patients & $0.1 \%$ & \\
\hline
\end{tabular}

The evidence shown in the Whole System Demonstrator Programme (Department of Health, 2011), the largest experiment of telemedicine held in England on 6,000 patients, agrees with our death rate reduction, hospitalization requests, and maintenance costs. 


\section{Second Scenario: Virtual Ward}

It conducted the development of a second line of experimental trial aiming to the creation of a virtual ward (Figure 4). It allowed developing a platform and an application to monitor activity of patient, to provide to nursing staff a tablet with the app. In the virtual ward outline, the goal was to monitor the whole ward at a glance in real time. The technological approach used for home monitoring was extended to non-intensive healthcare situations (Billis, Katzouris, Artikis, \& Bamidis, 2015; Eckermann \& Pekarsky, 2014).

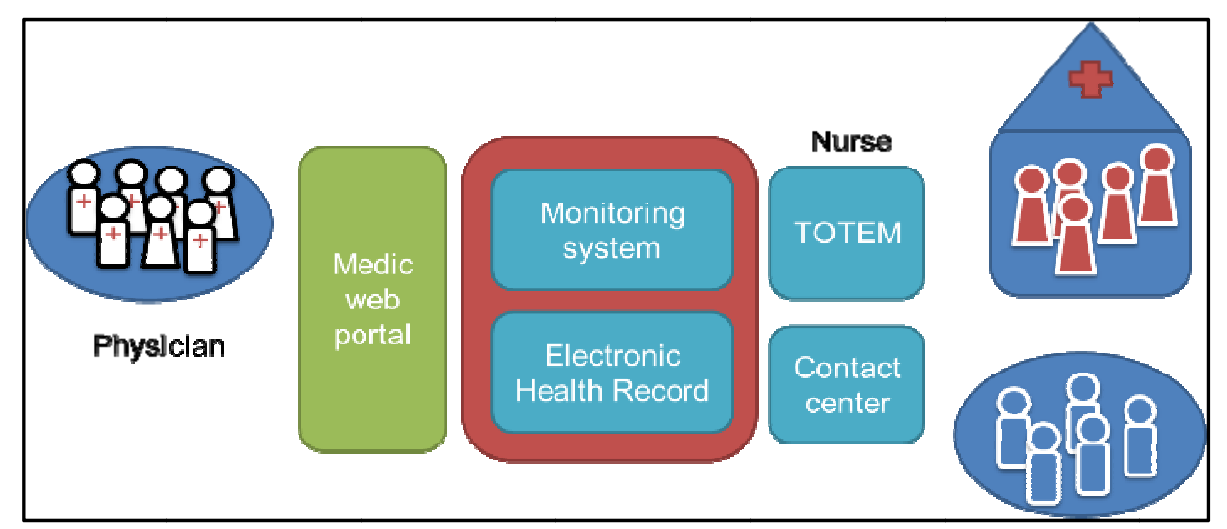

Figure 4. Functional model of the virtual ward implemented in.

The platform becomes a virtual and always accessible "communication window" between the management personnel and the medical staff at the clinical center (Konstantinidis, Antoniou, Bamparopoulos, \& Bamidis, 2014).

The nursing staff accesses to the common repository, exclusively for the part of their competence, through a common tablet and provides data entry directly in mobility, for example at the patient bed. Subsequently the nursing staff has the chance to examine the record inserted or to evaluate the medical history of a patient using a dedicated web portal.

The medical staff accesses to the full database that includes the part of the medical record of their medical competence. Such access is allowed through traditional web channels.

The modular design will allow future developments to add new classes of users such as administrative staff, staff of Healthcare Districts and family members of patients.

The sample of the patients involved in the experimentation consisted of 20 persons, equally divided in gender, and with an average age of 80.05 years.

The analysis of this other sample suggests a clear increase in the average age of the patients with respect to the one in the first scenario, with peaks of up to 93 years and a substantial balance with the gender of the participants. Unlike the patients monitored at home, in virtual ward there were no pre-established selection criteria regarding a specific disease because the realized technology provides its value in a transversal way regardless of the pathology of patient. During the analysis of the results of this experimental line, the dataset nursing was used to identify singularities (nursing record) or particular patterns occurred to the patient that could be indicators of potential future problems or exacerbations, looking for a corresponding event in the clinical dataset (medical records).

This research was carried out also in reverse, i.e. starting from the clinical dataset and looking for matches in the nursing one. Nevertheless, given the continuous intensity of patient care in the clinical center, the 
variations of these parameters from the norm are extremely connected to the medical staff and nurses in the emergency wards.

However, since all the patients have been already intensively monitored during the project as part of the regular assistive activity of the hospital, it was not possible to find significant variations of the clinical status of the patients. Nevertheless, in addition to logistical and organizational value, it is possible that the system, once adopted, will reveal important clinical findings.

The implemented system, used at full capacity, will allow: to increase efficiency in management of data on hospitalized patients; greater transparency and simplicity to access data for family members; a substantial contributes to the dematerialization of the current health record and thus saving costs.

This new system aroused also commercial interest among ICT producers for healthcare sector.

\section{Third Scenario: Evaluation in Rehabilitation}

The third line of experimentation is directed to patients with limited mobility who require rehabilitation treatments (Figure 5), because of their orthopedic and/or neurological issues.

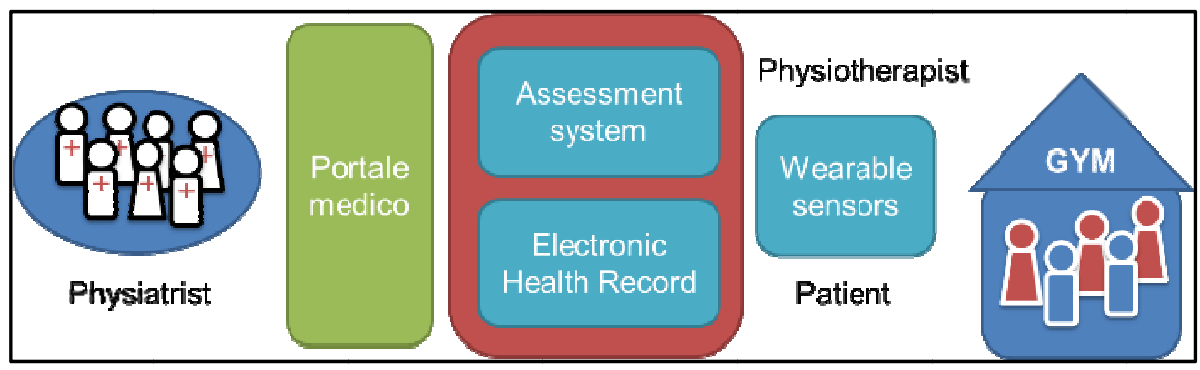

Figure 5. Functional model of the virtual rehabilitation.

The idea at the basis of this experiment starts from some clinical considerations linked to the current technique for the evaluation of patients. In fact, in case of clinical evaluation of disability with total or partial loss of ambulation there are two main types of possible patients involved: the Neurological and Orthopaedic. In the latter case, the parameters to be evaluated are represented by the oscillation of the basin, from the excursion of the hip, knee, and ankle, as well as other data of orthostatic nature. Starting with the physiological range of motion (R.O.M.) of the joints, standardized in relation to age and to possible surgery, it is possible to assess the objective articular recovery capacity and postural static-dynamic adjustments. It is therefore possible to identify the rehabilitation protocols and expected goals of the recovery with greater adherence to the real needs of the individual patient, and to check the clinical picture ab initio, in progress and at home.

The solution, selected on the basis of physiatrists' specs, has the possibility to integrate data directly into the technology repository already used for the other two experimental lines, but also includes an innovative system of measurement and data collection based on magnetoinertial wearable sensors (Moeslund et al., 2001). These sensors interface with a software that reproduces in a virtual reality environment, the same movements and the same R.O.M. variations that the patient carries out in real time.

The system also includes the possibility to perform a test called "six minute walking test (6MWT)". 6MWT allows measuring the functional residual capacity of a patient, and it is generally recommended for diagnostic purposes. 6MWT asked the patient to walk along a straight path for six minutes. After completing the test, it is estimated that the distance traveled will be the capacity motion index of the patient. In recent years, because of its simplicity of execution the test has aroused much interest and has proven to be adaptable to 
different situations. The implications of the execution of this test are relevant both from a clinical point of view and from a legal point of view. Shifting down the complexity of the problem, the attention was focused on analyzing the problem of rehabilitation of the lower limbs in patients with prostheses of hip and knee. Picking specifications directly from physiatrists, kinematic parameters were selected, mainly related to the excursion joint, to evaluate R.O.M. of the hip, knee, and ankle. In addition to this test, also the 6MWT was performed.

The basic idea of the experiment was to work alongside the experimental and the traditional protocols with the new measurements implemented with sensors. So, since on average a rehabilitation cycle lasts about 20 days, doctors decided to perform the technology mediated assessments on admission ( $\mathrm{t} 0$ ), a number of times during the rehabilitation period (t1, t2,..., tf-1) and at patient discharge (tf). The scientific goal of the project was to identify repeatable and quantitative indexes to characterize the quality of rehabilitation.

The final platform is composed of a set of small wearable wireless sensors anchored to elastic tearing bands or other anchoring structures on the body through a hanging mechanism (Patel et al., 2012). Each sensor has a unique code and is able to communicate with a receiver plugged to a PC through the USB port. The PC has an application interacting with the data from the central database already used for the other two lines of experimentation. The software allows selecting the patient, the test to perform, to calibrate the system, to perform the test, and to save the data in the same database.

A typical assessment session shows that the physician, the physical therapist, and the patient access the rehabilitation gym where the tests will be performed. The system is composed of a set of sensors (five or seven according to the need), one or more wireless USB receivers and a notebook with the application software installed.

In each evaluation session, a virtual evaluation board is compiled automatically or manually with the values of the test, the patient data, date and time, the sequence number of the test, the physiatrist/physiotherapist that is leading the test. The software consists of the following modules:

Initial interface. At the beginning of the session, the doctor needs to login to the system by inserting his personal credentials. Once logged in, the user selects the patient to be tested. In the case the patient is already present with previously acquired data, these can be displayed with the movement represented by the 3D manikin (replay). It includes a section in which you can select the test to perform. Also through this interface, you can replay acquisitions of tests carried out previously (Player) (Ward, 2013).

Calibration section. The left side of the screen displays a static figure of the manikin with the various points where to place sensors. Based on the labels present on the sensors, they will be placed in the appropriate holder of the manikin hardware. At the push of button "Default Calibration", each sensor is calibrated according to its default position. In case you wish to use the sensors in different positions or to other body segments, it will follow the traditional procedure provided by the software.

The test. The acquisition of R.O.M. occurs within a default time period (from $10 \mathrm{~s}$ to $60 \mathrm{~s}$ ) when the patient carries out angular movements of the joint autonomously or assisted by physiatrist. After completing the test, the software will record the entire route, as well as the maximum and minimum value reached. The 6MWT implies that the patient performs the test along a straight path (platform, corridor, etc.) of known length to go back and forth several times. The system will end the test automatically and calculate the number of steps taken (right and left), the average length of the steps, the speed of route and distance traveled. All the data are stored in the patient database. The test session save the stream of data in order to be able to playback later. The playback will present again the test with 3D animation and the possibility to start the movie, pause, restart from where you left off and restart it from the beginning. 


\section{Discussion}

\section{Consideration About New Paradigm}

End-User involvement: The living lab approach. The Living Lab is a new paradigm for industrial research and development allowing the final users (represented by a group of potential customers) to actively collaborate with the designers and technicians in the design, development and test of new products and services aimed to themselves. Living Labs stimulate social innovation by transferring research results from the closed industrial laboratory towards real life contexts where citizens and users become co-developers (Baedeker, Greiff, Grinewitschus, Hasselkuß, Keyson, Knutsson, Liedtke, Lockton, Lovric, Morrison, Van Rijn, Rohn, Silvester, Van Harinxma, \& Virdee, 2014). The end-user designs, with the help of engineers, the product of service according to his real needs, interest, and attitude. With Living Labs user communities are involved not only as observed subjects, but also as a source of creation.

This new approach is quickly getting popular in many international contexts, allowing, in particular small and medium-sized enterprises (SMEs) to create full-scale experiments in "pilot markets", thus improving the existing prototype and anticipating problems that may arise in the post-sales phase.

The Living Lab process is based on a continuous interaction of a multidisciplinary team, which repeats the following four main activities in a spiral way until they come to a final and satisfying solution:

1. Co-creation: bringing together technology push and application pull into a diversity of views, constraints, and knowledge sharing that sustains the ideation of new scenarios, concepts, and related artifacts. This phase can be considered as a sort of shared brainstorming.

2. Exploration: engaging all stakeholders, especially user communities, at the earlier stage of the co-creation process for discovering emerging scenarios, usages, and behaviors through live scenarios in real environments. This can be considered as a pre-experimental phase, in which the users imagine to test and validate the solutions identified in the co-creation phase.

3. Experimentation: implementing the proper level of technological artifacts to experience live scenarios with a large number of users while they are collecting data to be analyzed in their context during the evaluation activity.

4. Evaluation: assessing new ideas and innovative concepts as well as related technological artifacts in real life situations through various dimensions such as socio-ergonomic, socio-cognitive, and socio-economic aspects; making observations on the potentiality of a viral adoption of new concepts and related technological artifacts through a confrontation with users' value models.

Recently Apulia Region has started a new European funding program (European Regional Development Fund-ERDF) fostering the creation of new Living Labs focused on a set of different topics. Apulia Region first collected from local End-User Institutions and Associations the unsatisfied needs that could be addressed with suitable technology; then a call aimed at ICT Apulian SMEs for designing, prototyping, and testing new technological solutions together with the End Users and with the supervision of a Research Center has been issued.

End-users were involved using different modes of commitment, as well as for the involvement of citizenship. First of all medical and paramedical staff of the clinical center were involved, such as medical specialists and young interns, nurses, health workers, and physical therapists for a total of about 20 people. With the personnel involved, regular meetings were carried out at least once a week throughout the project: at 
the beginning the platforms specifications have been gathered, then the meetings served to check the progress of monitoring experience. In addition also GP who had charge of the selected patients was involved to collaborate in the patient monitoring. Regarding the other type of user end, all patients of the clinical center were sensitized with an appropriate communication campaign. In order to increase even more the resonance of the initiative information campaigns and communication were launched through the web and social networks: the PRO DOMO SUD web portal was made and the facebook page was created to post all the events and news.

Another method for the involvement of the population was to advertise the opening and the existence of the DemoLab at the high school and the nearby University of Taranto. Partly as a result of these activities, the Laboratory has hosted five young people in various capacities (internships, master, undergraduates).

DemoLab co-creation. At the end of experience, a DemoLab was created, a physical place where all citizens could come and test the solutions identified. The DemoLab was realized within the clinical center and it worked as an active laboratory in which not only the activities of the project were held, but was also used by other citizens, students and people outside to inform about technology and test in vivo the solutions implemented.

During the project five young students (a lawyer expert in intellectual property, a biomedical engineer, two undergraduates in psychology, and a degree in management engineering) found in the DemoLab the opportunity to further study and carry out a collaborative experience.

The DemoLab was also the reference center for all patients and relatives involved in the various experiments that during the year have asked for technical information and logistics. The DemoLab was also publicized in schools and the Faculty of Engineering of the nearby branch of the University of Bari in Taranto.

From Triple Helix to Quadruple Helix of development and innovation. The Triple Helix paradigm of development and innovation shows the correlation between the helices dynamics of "university, industry, government and relations" that drives knowledge and innovation in the glocal knowledge economy and society (Etzkowitz \& Loet, 2000). This project is similar to the model of the Quadruple Helix of the development and innovation paradigm where the "civil society" is described as the correlation between "media-based and culture-based public”. Knowledge and innovation policies and strategies must acknowledge the important role of the "public" for a successful achieving of goals and objectives. On the one hand, public reality is being constructed and communicated by the media and media system. On the other hand, the public is also influenced by culture and values. Knowledge and innovation policy should be inclined to reflect the dynamics of "media-based democracy," to draft policy strategies. For these reasons it is true the assumption that traditional economic policy gradually (partially) converts into innovation policy, leveraging knowledge for economic performance and thus linking the political system with the economy, then innovation policy should communicate its objectives and rationales, to the public, to seek legitimation (legitimacy) and justification (Colapinto \& Colin, 2012).

\section{Conclusion}

The management of age-related diseases is one of the most discussed and controverted topics in Healthcare policy: cost-effective solutions are needed to reduce the economic impact on welfare systems and to improve the quality of life of the patients. This is particularly true for chronic diseases, in which a simple, but continuous monitoring of suitable functional parameters able to evaluate the patient status would allow a consistent reduction of hospitalizations. 
In this paper the experience derived from a "Living Lab", a novel model of participated innovation in which the end-user plays a crucial role. It was codesigned, implemented, and tested m-health solutions for remote monitoring of physiological parameters directly from chronic patients' house according to a clinical protocol defined by healthcare professionals. For this aim set of parameters such as Blood Pressure, Body Weight, Heart Rate, Pulse Oximetry was measured and, if needed, the answers to a questionnaire. The proposed system allows reducing the hospitalization rate and increasing the empowerment of the patient in the self-management of his chronic health condition. Following the Guidelines on Telemedicine recently published by Italian Ministry of Health, the main results are the expression of a set of indicators being measured which are given below with their description.

(a) Size: volume of services provided;

(b) Continuity temporal duration and stability of service;

(c) Complexity: organizational complexity of service;

(d) Quality: standard performance and service response;

(e) Efficiency: cost of service;

(f) Effectiveness: comparison with the population of users affected and Telemedicine service;

(g) Customer Satisfaction by users (patients and caregivers).

The ICT platform was realized and tested for eight months on a sample of a selected chronic patients, recruited according a clinical protocol (i.e. number of hospitalization, age, proximity to the healthcare center) (Benvenuto, 2008). The trend of reduction of mortality, hospitalization, and cost is in line with the Whole System Demonstrator Project, the largest experiment of telemedicine held in England on 6,000 patients (BCC Research Healthcare Report, 2012). At the end of the trial, in compliance with the provisions of the Guidelines for Telemedicine, the patients were given a customer satisfaction questionnaire. The survey shows that the system has been considered easy to use and easy to learn and the same average patient declares that the service has improved its quality of life. Particularly interesting are the indications arising from the participation/interest of the general practitioner who was generally low and the increase of quality of life and health of the patient perceived. In support of the quality of such a systemic approach, the vice president of the EU recently underlined the experience of Scotland, which between 2006 to 2010 invested around 20 million Euros in the multidimensional management of chronic patients, obtaining benefits in terms of health and economic efficiency that have ensured almost three times the initial investment, reducing:

(a) emergency admissions by $20 \%$;

(b) hospital days by $14 \%$;

(c) prices by $8 \%$;

(d) the mortality rate for the chronically ill by $45 \%$.

It is therefore believed that such a multidimensional management approach may prove to be equally effective in the case of chronic patient management (Piscitelli, M. R. Giovagnoli, Bonifacino, Neglia, Benvenuto, Sambati, Giolli, \& A. Giovagnoli, 2015).

At the end of the trial, in compliance with the provisions of the Guidelines for Telemedicine, the patients were given a customer satisfaction questionnaire. The survey shows three important feedbacks: users considered the system easy to learn and easy to use; the service has improved the patients' quality of life. The experimental test of a remote monitoring platform showed how simple daily measurements and monitoring of physiological parameters performed autonomously by the patient at home and integrated with services of 
physiological support and customer care, could increase the patient's quality of life dramatically while reducing the public expenses for healthcare assistance. The most relevant result is that during the experimental test, no patient was hospitalized. This implies also about $€ 151,000$ of direct cost savings in public healthcare expenses. Considering the prevalence of cardiac failure and extending the projection to a larger number, the obtained economy could be consistent.

Another interesting outcome is the positive acceptability of the system, including the increase of life quality and healthcare assistance perceived (Benvenuto, Rizzo, \& Distante, 2008). All the patients were able to manage the smartphone and the medical devices with a short training period sometimes with the support of the call center. This is a first good result demonstrating that the co-design of the interface was effective and fully functional also considering the average age of the sample.

A negative aspect of the experience is the lack of interest or involvement by some healthcare professionals, especially GPs. This aspect must be taken into account and solved prior to extending the experimental experience as routine assistive service (Benvenuto, 2009).

It should be noticed that considering the limited amount of patients involved and the short-time interval of the experiment, all these results are purely preliminary indications that must be confirmed by more experimental data.

\section{References}

BCC Research Healthcare Report. (2012). Global markets for telemedicine technologies. Report ID: HLC014E.

Baedeker, C., Greiff, K., Grinewitschus, V., Hasselkuß, M., Keyson, D., Knutsson, J., Liedtke, C., Lockton, D., Lovric, T., Morrison, G., Van Rijn, M., Rohn, H., Silvester, S., Van Harinxma, W., \& Virdee, L. (2014). Proceeding from the 5th International Sustainable Transitions (IST) Conference, Utrecht, The Netherlands, August 27-29, 2014: Transition Through Sustainable Product and Service Innovations in Sustainable Living Labs: Application of User-centred Research Methodology within four Living Labs in Northern Europe.

Basilakis, J., Lovell, N. H., Redmond, S. J., \& Celler, B. G. (2010). Design of a decision-support architecture for management of remotely monitored patients. IEEE Engineering in Medicine and Biology Society, 14(5), 1216-1226. doi: 10.1109/TITB.2010.2055881

Benvenuto, M. (2008). I sistemi informativi per la gestione integrata ed ottimizzata delle risorse nel campo della malattie cardiovascolari. Università di Pisa.

Benvenuto, M., Rizzo, V., \& Distante, A. (2008). Assistenza primaria: modello innovativo per la salute (pp. 44-46). Tecnica Ospedaliera. Ed. Tecniche Nuove.

Benvenuto, M. (2009). La primary care con particolare attenzione al ruolo della medicina di base per l'efficienza $e$ appropriatezza prescrittiva. Il Sistema sanitario della Basilicata nel 2008. Una risorsa qualificante in uno scenario in cambiamento (pp. 129-145). The McGraw-Hill Companies.

Billis, A. S., Papageorgiou, E. I., Frantzidis, C. A., Tsatali, M. S., Tsolaki, A. C., \& Bamidis, P. D. (2015). A decision-support framework for promoting independent living and ageing well. IEEE Journal of Biomedical and Health Informatics, 19, 199-209. doi: 10.1109/JBHI.2014.2336757

Billis, A. S., Katzouris, N., Artikis, A., \& Bamidis, P. D. (2015). Clinical decision support for active and healthy ageing: An intelligent monitoring approach of daily living activities. In F. Pereira, P. Machado, E. Costa, and A. Cardoso (Eds.). EPIA 2015. LNCS (LNAI), Springer, 128-133. Retrieved from: http://link.springer.com/chapter/10.1007/978-3-319-23485-4_61

Bloom, D., Cafiero, E., Jané-Llopis, E., Abrahams-Gessel, S., Bloom, L., Fathima S., Feigl, A., Gaziano, T., Hamandi, A., Mowafi, M., O’Farrell, D., Ozaltin, E., Pandya, A., Prettner, K., Rosenberg, L., Seligman, B., Stein, A., Weinstein, C., \& Weiss, J. (2012). The global economic burden of noncommunicable diseases. PGDA Working Papers, Program on the Global Demography of Aging.

Clark, R. A., Conway, A., Poulsen, V., Keech, W., Tirimacco, R., \& Tideman, P. (2013). Alternative models of cardiac rehabilitation: A systematic review. European Journal of Preventive Cardiology, 22(1), 35-74. doi: $10.1177 / 2047487313501093$ 
Claxton, K., Paulden, M., Gravelle, H., Brouwer, W., \& Culyer, A. J. (2011). Discounting and decision making in the economic evaluation of health-care technologies. Health Economics, 20(1), 2-15. doi: 10.1002/hec.1612

Colapinto, C., \& Colin, P. (2012). Innovation in creative industries: From the quadruple helix model to the systems theory. Journal of the Knowledge Economy, 3, 343-353. doi: 10.1007/s13132-011-0051-x

Cunningham, P. J. (2010). The growing financial burden of health care: National and state trends, 2001-2006. Health Affairs, 1-8. doi: 10.1377/hlthaff.2009.0493

Department of Health. (2011). Whole system demonstrator programme-Headline findings.

Devlin, N., \& Parkin, D. (2004). Does NICE have a cost-effectiveness threshold and what other factors influence its decisions? A binary choice analysis. Health Economics, 13(5), 437-452. doi: 10.1002/hec.864

Eckermann, S., \& Pekarsky, B. (2014). Can the real opportunity cost stand up: Displaced services, the straw man outside the room. Pharmacoeconomics, 32, 319-325. doi: 10.1007/s40273-014-0140-3

Etzkowitz, H., \& Loet, L. (2000). The dynamics of innovation: From national systems and "Mode 2" to a Triple Helix of university-industry-government relations. Research Policy, 29(2), 109-123. doi: 10.1016/S0048-7333(99)00055-4

Fried, H. O., Lovell, C. A. K., \& Schmidtet, S. S. (1993). The measurement of productive efficiency: Techniques and applications. Oxford University Press.

Kakadiaris, I., \& Metaxas, D. (2000). Model-based estimation of 3D human motion. IEEE Transactions on Pattern Analysis and Machine Intelligence, 22(12), 1453-1459. doi: 10.1109/34.895978

Konstantinidis, E. I., Antoniou, P. E., Bamparopoulos, G., \& Bamidis, P. D. (2014). A lightweight framework for transparent cross platform communication of controller data in ambient assisted living environments. Information Sciences, 300, 124-139. doi: 10.1016/j.ins.2014.10.070

Lovell, N., Redmond, S. J., Basilakis, J., Shany, T., \& Celler, B. G. (2010). Telehealth technologies for managing chronic disease-Experiences from Australia and the UK. IEEE Engineering in Medicine and Biology Society, 5267-5269. doi: 10.1109/IEMBS.2010.5626312.

Marcin, J. P., Nesbitt, T. S., Cole, S. L., Knuttel, R. M., Hilty, D. M., Prescott, P. T., \& Daschbach, M. M. (2005). Changes in diagnosis, treatment, and clinical improvement among patients receiving telemedicine consultations. Telemedicine and e-Health, 11, 36-43. doi: 10.1089/tmj.2005.11.36

Ministero della Salute. (2014). Linee guida di indirizzo nazionali sulla Telemedicina, 2014.

Moeslund, T. B., \& Granumet, E. (2001). A survey of computer vision based human motion capture. Computer Vision and Image Understanding, 81(3), 231-268. doi: 10.1006/cviu.2000.0897

Pacht, E. R., Turner, J. W., Gailiun, M., Violi, L. A., Ralston, D., Mekhjian, H. S., \& St John, R. C. (1998). Effectiveness of telemedicine in the outpatient pulmonary clinic. Telemedicine Journal, 4, 287-292. doi: 10.1089/tmj.2005.11.36

Patel, S., Park, H., Paolo, B., Chan, L., \& Rodgers, M. (2012). A review of wearable sensors and systems with application in rehabilitation. Journal of Neuro Engineering and Rehabilitation, 9, 21. doi: 10.1186/1743-0003-9-21

Paulden, M., \& Claxton, K. (2012). Budget allocation and the revealed social rate of time preference for health. Health Economics, 21, 612-618. doi: 10.1002/hec.1730

Pinnock, H., Hanley, J., Lewis, S., Mac Need, W., Pagliarie, C., Van Der Polf, M., Sheikhg, A., \& McKinstryh, B. (2010). The impact of a telemetric chronic obstructive pulmonary disease monitoring service: Randomised controlled trial with economic evaluation and nested qualitative study. Primary Care Respiratory Journal, 18(3), 233-235. doi: 10.4104/pcrj.2009.00040

Piscitelli, P., Giovagnoli, M. R., Bonifacino, A., Neglia, C., Benvenuto, M., Sambati, F. V., Giolli, L., \& Giovagnoli, A. (2015). Diagnostic and therapeutic path of breast cancer: Effectiveness, appropriateness, and costs-results from the DOCMa study. Clinical Interventions in Aging, 10, 741-749. doi: 10.2147/CIA.S75486

Simoens, S. (2010). How to assess the value of medicines? Frontiers in Pharmacology, 1(115), 1-9. doi: 10.3389/fphar.2010.00115

Ward, R. (2013). The application of technology acceptance and diffusion of innovation models in healthcare informatics. Health Policy and Technology, 2(4), 222-228. doi: 10.1016/j.hlpt.2013.07.002

Weinstein, R. S., Lopez, A. M., Joseph, B. A., Erps, K. A., Holcomb, M., Barker, G. P., \& Krupinski, E. A. (2014). Telemedicine, telehealth, and mobile health applications that work: Opportunities and barriers. The American Journal of Medicine, 127(3), 183-187. doi:10.1016/j.amjmed.2013.09.032

WHO. (2010). Telemedicine: Opportunities and developments in Member States. Report on the second global survey on eHealth-Global Observatory for eHealth series, 2.

Zanaboni, P., \& Wootton, R. (2012). Adoption of telemedicine: From pilot stage to routine delivery. BMC Medical Informatics and Decision Making, 12(1), 1-9. doi: 10.1186/1472-6947-12-1 